\title{
Development of a model to predict pathologic response to chemotherapy in patients with colorectal liver metastases
}

\author{
Da Xu", Yan-Yan Wang", Xiao-Luan Yan, Juan Li, Kun Wang, Bao-Cai Xing \\ Hepatopancreatobiliary Surgery Department I, Key Laboratory of Carcinogenesis and Translational Research, Ministry of Education, Peking \\ University School of Oncology, Beijing Cancer Hospital and Institute, Beijing, China \\ Contributions: (I) Conception and design: BC Xing, D Xu, YY Wang; (II) Administrative support: BC Xing, K Wang; (III) Provision of study materials \\ or patients: D Xu, XL Yan, J Li; (IV) Collection and assembly of data: D Xu, XL Yan, J Li; (V) Data analysis and interpretation: D Xu, YY Wang; (VI) \\ Manuscript writing: All authors; (VII) Final approval of manuscript: All authors. \\ \#These authors contributed equally to this work. \\ Correspondence to: Bao-Cai Xing, MD. Hepatopancreatobiliary Surgery Department I, Key Laboratory of Carcinogenesis and Translational Research, \\ Ministry of Education, Peking University School of Oncology, Beijing Cancer Hospital and Institute, 52 Fucheng Road, Haidian District, Beijing \\ 100142, China. Email: xingbaocai88@sina.com.
}

Background: Preoperative chemotherapy has widely been used in colorectal cancer liver metastasis
(CRLM). Pathological response to chemotherapy is very important in evaluating tumor biology. However,
there is still a lack of a non-invasive and accurate method to evaluate pathological response before surgery.
Methods: We retrospectively analyzed the clinicopathologic data of patients with CRLM who underwent
liver resection after preoperative chemotherapy between January 2006 and December 2018. Pathological
responses were defined as minor when there are $\geq 50 \%$ remnant viable cells and as major when $0-49 \%$
remnant viable cells exist.

Results: A total of 482 patients were included and randomly divided into training $(\mathrm{n}=241)$ and validation $(n=241)$ cohorts. The proportion of major pathologic response was similar between the two groups $(51.5 \%$ and $48.5 \%$ ). Multivariate analysis determined the disease-free interval (DFI), tumor size, tumor number, and RAS status as independent predictors of major pathologic response to preoperative chemotherapy. The nomogram incorporating these variables showed good concordance statistics in the training cohort $(0.746$, 95\% CI: $0.685-0.807)$ and validation cohort (0.764, 95\% CI: 0.704-0.823). In addition, the nomogram showed good applicability in patients with different characteristics.

Conclusions: The established nomogram model performed well in predicting pathological response in patients with CRLM.

Keywords: Colorectal liver metastasis; pathologic response; chemotherapy; predictive model

Submitted Feb 08, 2021. Accepted for publication Jun 08, 2021.

doi: 10.21037/jgo-21-82

View this article at: https://dx.doi.org/10.21037/jgo-21-82

\section{Introduction}

Surgical resection is currently the most effective treatment for colorectal cancer liver metastasis (CRLM), with a 5 -year survival rate of $40-50 \%$ (1). Preoperative chemotherapy has been widely used in CRLM. Although the benefit in prolonging survival has long been controversial, the effect of shrinking tumor to allow for parenchymal sparing hepatectomy (PSH), controlling the micro-metastatic disease, and testing tumor biology by assessing chemotherapy response has been widely accepted (2-4). Chemotherapy response is a very strong predictor of long-term survival. Patients who present good response in preoperative chemotherapy show significantly better survival compared with those who had poor response after hepatectomy (5). For those who show poor response, a more 
effective regimen might be used rather than performing surgery directly. Thus, it is very important to evaluate the chemotherapy response with high accuracy (6).

Response evaluation criteria in solid tumors (RECIST) has long been used to evaluate chemotherapy response based on the tumor size change, however, it can be rather inaccurate in assessing tumor size change in patients with irregular tumor shapes (7). The most accurate way to evaluate true chemotherapy response is pathologic examination, especially for patients treated with targeted drugs who are often presented as composition changes rather than size change $(8,9)$. By assessing the residual tumor cell percentage, we can objectively assess the true response status. Precisely testing the pathologic response is very helpful in deciding on the most optimal timing to perform the surgery and knowing the prognosis of patients undergoing surgery (6). However, a pathologic response could hardly be obtained before the surgery without resected specimens. Preoperative biopsy can be invasive and not accurate enough because of the tumor heterogeneity. Recently, several studies found that some clinical factors are associated with pathologic response, including $R A S$ gene status, chemotherapy regimens and clinical characteristics $(10,11)$. The aim of this study is to develop and validate a model to accurately predict pathologic response to chemotherapy in a non-invasive way.

We present the following article in accordance with the TRIPOD reporting checklist. Available at https://dx.doi. org/10.21037/jgo-21-82.

\section{Methods}

The study was conducted in accordance with the Declaration of Helsinki (as revised in 2013). The study was approved by Ethical Review Board committee of the Beijing Cancer Hospital and Institute (NO. 2015KT32) and informed consent was taken from all the patients.

\section{Study design}

Pathologically confirmed CRLM patients after preoperative chemotherapy following liver resection between January 2006 and December 2018 in the HPB Surgery Ward I at the Beijing Cancer Hospital (Beijing, China) were retrospectively identified from our CRLM database. Exclusion criteria were patients who: (I) underwent direct surgery; (II) underwent palliative surgery; (III) having no pathologic response information; (IV) with missing clinical information; (V) being lost to follow-up.

\section{Assessment of pathologic response following chemotherapy}

All CRLM tumors of included patients were sampled and the hematoxylin \& eosin stained sections were reviewed by gastrointestinal pathologists. Pathologic response to chemotherapy was categorized according to MD Anderson group (8). The TRG results was independently reviewed by two senior pathologists. Major response was defined as from $1 \%$ to $49 \%$ residual cancer cells, and minor response as $\geq 50 \%$ residual cancer cells remaining. For patients with multiple liver metastases, the TRG value was calculated using the average TRG value of the largest 3 lesions in order to reduce the heterogeneity between each CRLM tumors.

\section{Perioperative management and follow-up}

Multidisciplinary team meetings were scheduled weekly in our center for patients with CRLM. Gene status was assessed in every patient and included at least the following: KRAS (codons 12, 13 and 61); NRAS (codons 12, 13 and 61); and $B R A F$ (v600e). Contrast-enhanced MRI combined with diffusion-weighted MRI were routinely performed before and every 2-4 cycles after chemotherapy. Chest and pelvic CT scans were routinely performed. Positron emission tomography-CT scans were not routinely performed unless patients were suspected as having extrahepatic disease. The criteria to schedule for neoadjuvant chemotherapy for patients with CRLM mainly include those: with high risk of clinical risk scores (A scoring system based on 5 factors: node-positive primary, disease-free interval (DFI) of CLM $<12$ months, CRLM number $>1$, largest CRLM $>5 \mathrm{~cm}$, and carcinoembryonic antigen (CEA) $>200 \mathrm{mg} / \mathrm{L}$, each scoring 1. A score of 0-2 were considered low risk group, and 3-5 were considered high-risk group), with multiple liver metastases, develop early liver metastases during adjuvant chemotherapy of primary tumor, liver metastases was technically difficult to resect. Neoadjuvant chemotherapy includes oxaliplatin- or irinotecan-based chemotherapy (mFOLFOX6/XELOX/FOLFIRI/FOLFOXIRI) regimens. When the patients have heavier tumor burden or technically difficult to resect, targeted agents (Bevacizumab and Cetuximab) or even in combination with triplet chemotherapy would be used. The time interval between the date of the last chemotherapy session and hepatic surgery was usually $2-4$ weeks, extending to $6-8$ weeks with 
the addition of bevacizumab. Primary tumors located in the cecum, ascending colon, and transverse colon were defined as right side (RS) tumors, and those located in the splenic flexure, descending colon, sigmoid colon, and rectum were defined as left side (LS) tumors. Contrast-enhanced CT scans or MRI, liver function tests, and measurements of carcinoembryonic antigen levels were performed every 3 months after the surgery. For patients with liver-limited recurrence, the treatment option of choice was localized treatment, including surgical or ablation techniques.

\section{Patient selection for liver resection and operative technique}

For some patients, the primary tumor was resected at another institution. The surgical treatment of all LMs was conducted at our center. Resectable was defined as complete remove of macroscopic tumors, while maintaining at least a $30 \%$ future liver remnant (FLR) or a remnant liver to body weight ratio $>0.5$ with sufficient blood inflow and outflow of the liver (6). Intra-operative ultrasound was routinely performed during the hepatectomy to detect the presence of any further or yet undetected lesions.

\section{Statistical analyses}

Categorical variables were presented as numbers with percentages and compared using the Chi-squared test. Variables that were statistically significant in the univariate analysis were included in the multivariable logistic regression analysis to determine independent predictors of major pathologic response. A nomogram was formulated based on the results of multivariate logistic regression analysis. The predictive performance of the nomogram was measured by concordance (C) statistics and calibration plots. In subgroup analysis, receiver operating characteristic (ROC) curve analysis was conducted, and the area under the ROC curve (AUC) was calculated to further evaluate the predictive performance of the nomogram. All statistical analyses were conducted using SPSS software version 24.0 (IBM Corp., Armonk, NY, USA) and R version 3.5.1 (http://www.r-project.org/). A $\mathrm{P}<0.05$ was considered statistically significant.

\section{Results}

\section{Characteristics of patients}

A total of 932 patients underwent hepatectomy between
January 2006 and December 2018 in our center. Six hundred and fourteen patients received preoperative chemotherapy, and 132 patients was excluded according to the exclusion criteria. Finally, a total of 482 patients met the inclusion criteria and were enrolled in this study. The included 482 patients were randomly divided into the training cohort $(\mathrm{n}=241)$ and validation cohort $(n=241)$. The baseline characteristics of patients in the training cohort and validation cohort are summarized in Table 1. Baseline data were similar between the training cohort and validation cohort. The median number of preoperative chemotherapy cycles were 4 for both the training cohort and validation cohort. Major pathologic response was observed in $241(50.0 \%)$ of the total patients, including 29 patients with complete pathologic responses $(6.0 \%)$. The proportion of major pathologic response was similar between the training cohort and validation cohort $(51.5 \%$ vs. $48.5 \%, \mathrm{P}=0.524)$. Based on radiographic assessment, tumor decrease was observed in $396(82.2 \%)$ of the total patients. For patients with tumor increase after preoperative chemotherapy, major pathologic response was observed in $19(42.2 \%)$ and 20 $(48.8 \%)$ patients in the training cohort and validation cohort, respectively. According to the RECIST criteria, complete or partial response was observed in 223 (46.1\%) of the total patients.

\section{Predictors of major pathologic response in the training cohort}

In the univariate logistic regression analysis, DFI, tumor size, tumor number, RAS mutational status, and CEA level were identified as predictors of major pathologic response to preoperative chemotherapy (Table 2). The above factors were included in the multivariable logistic regression analysis. Multivariate analysis determined that DFI $[<12 v s$. $\geq 12$ months, OR (95\% CI): 1.908 (1.026-3.547), $\mathrm{P}=0.041$ ], tumor size [ $<3$ vs. $\geq 3 \mathrm{~cm}$, OR (95\% CI): 5.076 (2.849-9.010), $\mathrm{P}<0.001$ ], tumor number [single $v$ s. multiple, OR (95\% CI): 2.625 (1.302-5.291), $\mathrm{P}=0.007$ ], and $R A S$ status [wild-type $v s$. mutated, OR (95\% CI): 2.217 (1.227-4.000), $\mathrm{P}=0.008$ ] were identified as independent predictors of major pathologic response to preoperative chemotherapy (Table 2).

\section{Development and validation of a nomogram for predicting major pathologic response}

The above four independent predictors of major pathologic 
Table 1 Baseline characteristics

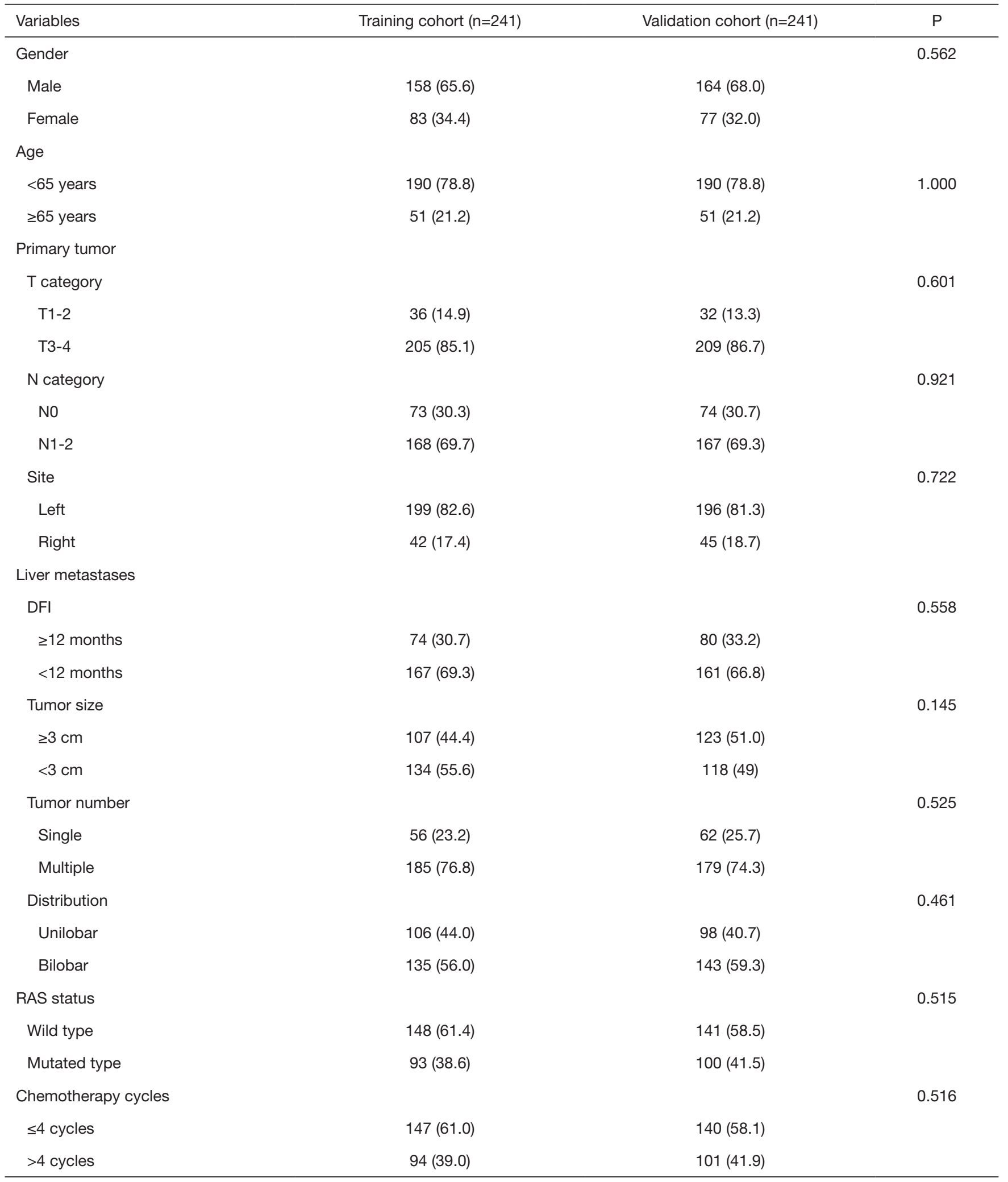

Table 1 (continued) 
Table 1 (continued)

\begin{tabular}{|c|c|c|c|}
\hline Variables & Training cohort $(n=241)$ & Validation cohort $(n=241)$ & $\mathrm{P}$ \\
\hline No & 167 (69.3) & $154(63.9)$ & \\
\hline Yes & $74(30.7)$ & $87(36.1)$ & \\
\hline CEA & & & 0.016 \\
\hline$>50 \mathrm{ng} / \mathrm{mL}$ & $59(24.5)$ & $83(34.4)$ & \\
\hline Pathologic response & & & 0.524 \\
\hline Minor & $117(48.5)$ & $124(51.5)$ & \\
\hline Major & $124(51.5)$ & $117(48.5)$ & \\
\hline Minor pathologic response & $91(46.4)$ & $103(51.5)$ & \\
\hline Major pathologic response & $105(53.5)$ & $97(48.5)$ & \\
\hline Increase & $45(18.7)$ & $41(17.0)$ & \\
\hline Minor pathologic response & $26(57.8)$ & $21(51.2)$ & \\
\hline Major pathologic response & $19(42.2)$ & $20(48.8)$ & \\
\hline RECIST assessment & & & 0.784 \\
\hline Complete or partial response & $113(46.9)$ & $110(45.6)$ & \\
\hline Stable or progressive disease & $128(53.1)$ & $131(54.4)$ & \\
\hline
\end{tabular}

*, Evaluated based on imaging examinations. Decrease, reduction in the sum of target lesion diameters; Increase, increased in the sum of target lesion diameters. CEA, carcinoembryonic antigen; DFI, disease-free interval; RECIST, Response Evaluation Criteria in Solid Tumors.

response were integrated into a major pathologic response estimation nomogram (Figure 1). The c-statistic of the nomogram in discriminating major pathologic response in the training cohort was 0.746 (95\% CI: $0.685-0.807)$, and the calibration plot showed good agreement between the predicted probability of major response and the observed incidence of major response (Figure $2 A$ ). In the validation cohort, the $\mathrm{C}$ statistic of the nomogram was 0.764 (95\% CI: 0.704-0.823), and the calibration plot also showed good agreement between predictions and actual observations (Figure $2 B$ ). The optimized cutoff of the nomogram score for predicting major pathologic response was 124 . With this optimized cutoff, the sensitivity and specificity of the nomogram for predicting major pathologic response were $75.0 \%$ and $65.0 \%$ in the training cohort and $66.7 \%$ and $71.0 \%$ in the validation cohort.

\section{Subgroup analysis}

To evaluate the applicability of the nomogram in patients with different characteristics, subgroup analyses were performed based on primary tumor site (left or right colon), RAS status (wild-type or mutated), and the use of bevacizumab (yes or no). In each subgroup, the nomogram performed well in predicting major pathologic response to preoperative chemotherapy (all AUCs $>0.700$, Figure $3 A, B, C, D, E, F)$.

\section{Discussion}

Many previous studies have tried to predict pathologic response to chemotherapy. However, a comprehensive model for predicting pathologic response (10-13) is still lacking. This study established a prognostic model to 
Table 2 Uni- and multivariable analyses to identify predictors of major pathologic response in the training cohort

\begin{tabular}{|c|c|c|c|c|c|c|}
\hline Variables & \multicolumn{3}{|c|}{ Univariable } & \multicolumn{3}{|c|}{ Multivariable } \\
\hline Gender (male vs. female) & 0.784 & $0.460-1.337$ & 0.372 & - & - & - \\
\hline Age ( $\geq 65$ vs. $<65$ years) & 0.800 & $0.431-1.486$ & 0.480 & - & - & - \\
\hline \multicolumn{7}{|l|}{ Primary tumor } \\
\hline $\mathrm{N}$ category (N1-2 vs. N0) & 1.045 & $0.603-1.811$ & 0.875 & - & - & - \\
\hline Site (right vs. left) & 0.740 & $0.379-1.443$ & 0.376 & - & - & - \\
\hline \multicolumn{7}{|l|}{ Liver metastases } \\
\hline DFI (<12 vs. $\geq 12$ months) & 1.889 & $1.083-3.292$ & 0.025 & 1.908 & $1.026-3.547$ & 0.041 \\
\hline Distribution (unilobar vs. bilobar) & 1.447 & $0.867-2.415$ & 0.157 & - & - & - \\
\hline RAS status (wild type vs. mutated type) & 1.739 & $1.029-2.932$ & 0.038 & 2.217 & $1.227-4.000$ & 0.008 \\
\hline Chemotherapy cycles ( $\leq 4$ vs. $>4$ ) & 1.563 & $0.928-2.632$ & 0.093 & - & - & - \\
\hline Bevacizumab (yes vs. no) & 1.162 & $0.672-2.012$ & 0.591 & - & - & - \\
\hline CEA ( $\leq 50$ vs. $>50$ ng/mL) & 2.597 & $1.406-4.808$ & 0.002 & 1.684 & $0.833-3.401$ & 0.147 \\
\hline Tumor radiographic assessment (decrease vs. increase) & 1.580 & $0.820-3.040$ & 0.172 & - & - & - \\
\hline
\end{tabular}

CEA, carcinoembryonic antigen; DFI, disease-free interval; OR, odds ratio.

predict pathologic response after chemotherapy based on several clinical factors that are obtainable in a noninvasive fashion. The weight and correlation of each factor influencing pathologic response was fully considered. The validation results suggest that the model could predict pathologic response with precision, as well as good sensitivity and specificity.

The RECIST criteria are currently widely used to evaluate chemotherapy response based on radiographic tumor change. As systematic treatment protocols and targeted drugs are refined, tumor response would also present as internal composition changes. Tumor tissue was replaced by necrosis and inflammatory cells in pathologic examination, causing tumor size to remain stable or even increase. Previous studies shown that although some patients were evaluated as unresponsive by RECIST criteria, major response could still be found by pathological examinations (14). Consistently, in this study, major pathologic response was also observed in $39(45.3 \%)$ patients of those with tumor increase after preoperative chemotherapy. This finding indicates that tumor radiographic changes after chemotherapy are not completely in consistent with the pathological response. Hence, the model for predicting pathological response in this study could be considered as a supplement to evaluate chemotherapy response and helps to make treatment strategies more comprehensively. For patients who were predicted to show major pathologic response despite stable tumor size, hepatectomy could be performed if the liver metastasis was resectable. If the metastases were still unresectable, our nomogram could allow for continuing with chemotherapy in pursuit of maximum pathologic response, and even complete pathologic response $(15,16)$. In addition, patients with major pathologic response would show clearer tumor-normal liver interface (TNI) $(17,18)$, allowing for narrower or even R1 margins to be acceptable during surgery (19). However, if patients were predicted to have minor pathologic response and still stable or progressing in size, it would be more reasonable to select a second-line chemotherapy regimen with higher efficiency to control tumor growth instead of pursuing surgery $(4,20)$.

In this study, we found several clinical factors that 


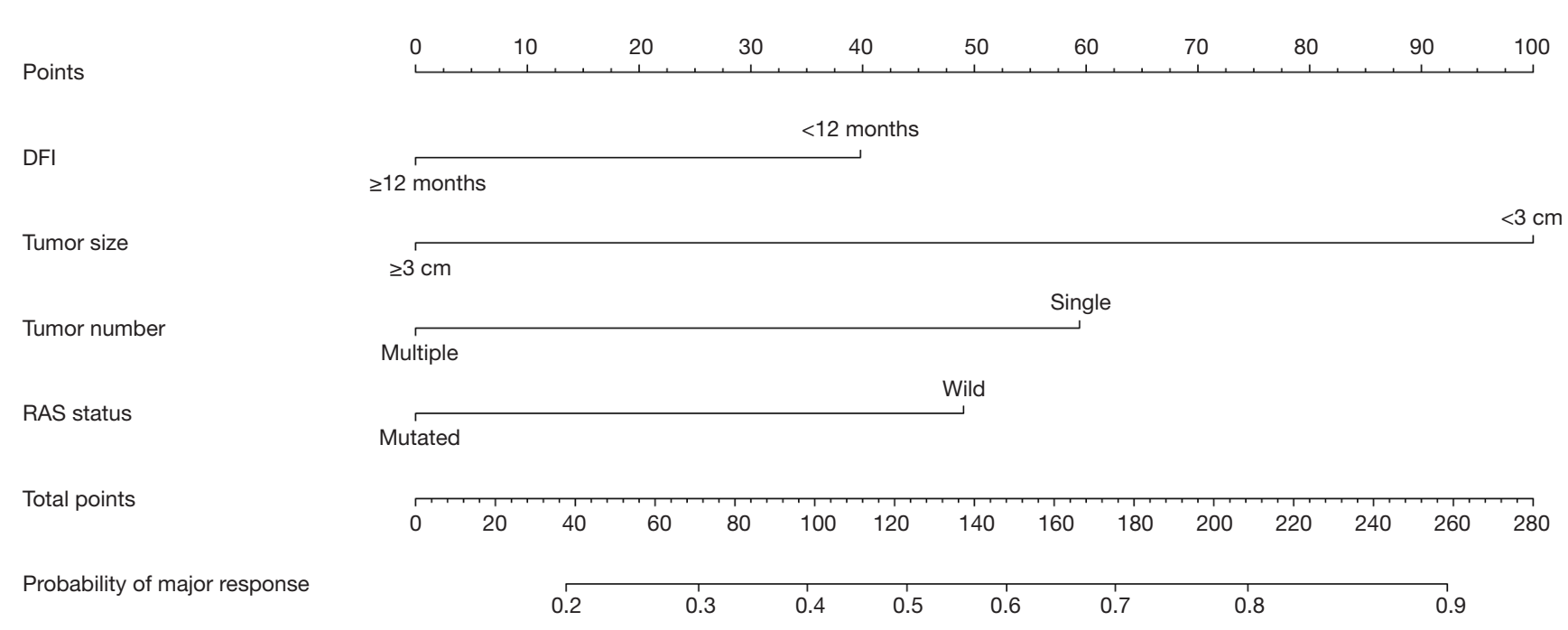

Figure 1 Nomogram for predicting major pathologic response to preoperative chemotherapy in patients with CRLM. DFI, disease-free interval.
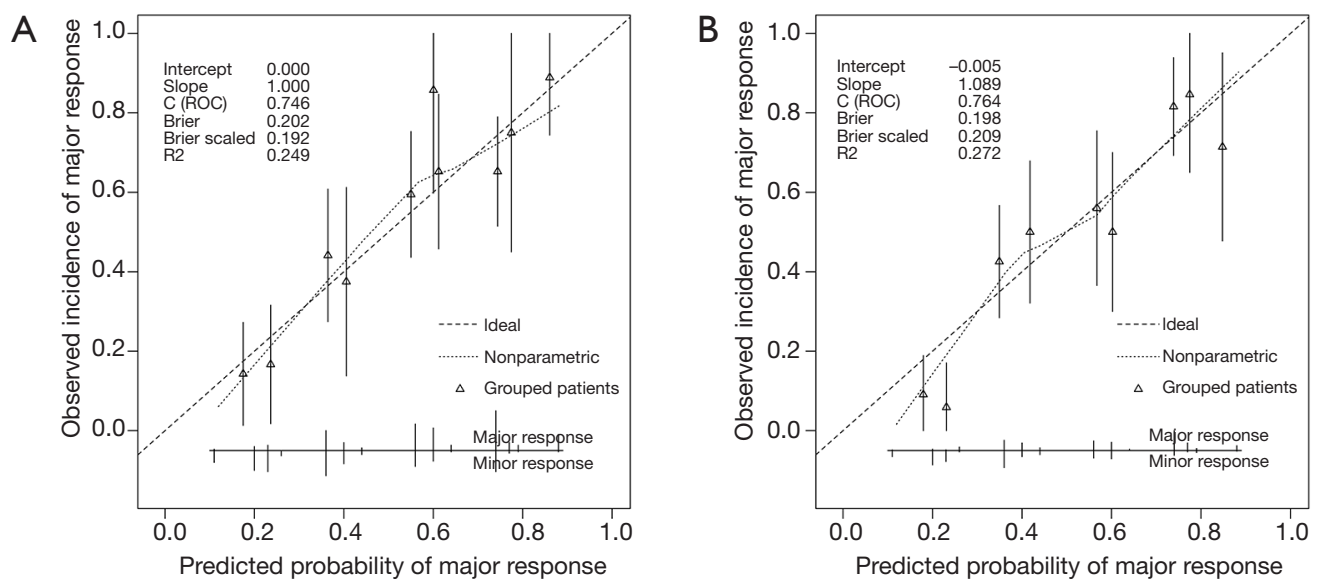

Figure 2 Calibration plots of the observed incidence versus predicted probability of major pathologic response of the nomogram. (A) In the training cohort; (B) In the validation cohort.

predicted the pathologic response of patients with CRLM to preoperative chemotherapy. Patients with $R A S$ wildtype were more likely to experience a major response after preoperative chemotherapy. $R A S$ status has long been known to be associated with long-term survival and the sensitivity to chemotherapy $(21,22)$. Previous studies also found similar results showing that major responses were more common in patients with $R A S$ wild-type compared with those with $R A S$ mutant $(10,13)$. Tumor size and tumor number has also been proven to correlate with degree of pathologic response. Zimmitti et al. and
Margonis et al. showed that CRLM size $>3 \mathrm{~cm}$ was an independent predictor of poor response to chemotherapy $(10,13)$. Patients with larger tumors and higher tumor number reflected a heavier tumor burden, which is already known to be a negative factor for survival (23). DFI $<12$ months is another factor shown to be in association with major pathological response. It might be explained by the following reason. Patients with DFI $>12$ months who receive neoadjuvant chemotherapy might have heavier tumor burden, and less likely to show major response, leading to the probably of selection bias. This is in line with 

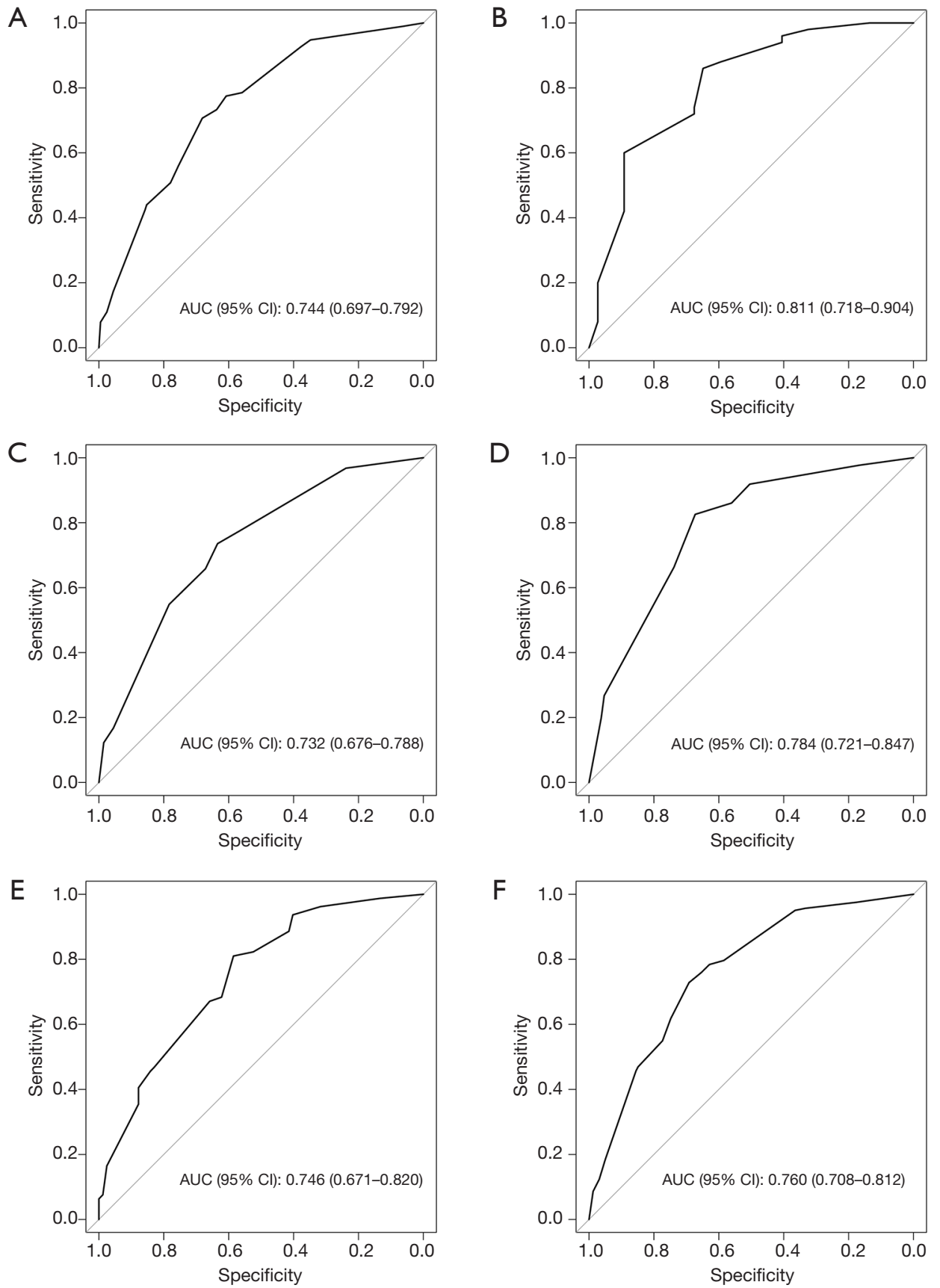

Figure 3 Validity of the nomogram performance in predicting major pathologic response in patients with different characteristics. (A) Primary tumor located in left-side; (B) Primary tumor located in right-side; (C) RAS wild type; (D) RAS mutated type; (E) Treated with bevacizumab; (F) Treated without bevacizumab.

our conclusion that patients with limited liver metastases are easier to show major pathologic response. Besides, patients with metachronous liver metastases (DFI $\geq 12$ months) might have received adjuvant chemotherapy after resection of primary tumors. Therefore, it is often needed to change to 2nd-line chemotherapy when liver metastases occur. Previous literature shown that the efficiency of secondline chemotherapy was only about $20 \%$ (24), so it might be difficult for these patients to develop major pathologic response. However, patients with simultaneous liver 
metastases usually don't receive any treatment previously, so their pathologic response after preoperative chemotherapy would be relatively higher.

It was interesting that some other factors, such as primary tumor site and the use of bevacizumab $(11,25)$, which were previously shown to be related to chemotherapy response, were found not to be independent factors influencing pathologic response in our study. Margonis et al. also found that adding bevacizumab was not associated with pathologic response in multivariate analysis $(\mathrm{P}=0.3)(10)$. Primary tumor location was also an important factor for chemotherapy response. Serayssol et al. and Wang et al. found that it was associated with poor pathologic response $(11,12)$. However, other studies did not find similar results. In order to avoid bias arising from to patient selection and operating within a single-center sample, we choose three important factors-primary tumor site, $R A S$ mutational status, and the use of bevacizumab and performed a subgroup analysis to assess the predictive power of this model in different subgroups. The results showed that the nomogram performed well in predicting major pathologic response to preoperative chemotherapy in patients with different characteristics.

There are several limitations in this study. The main disadvantage of this study is that it uses data collected retrospectively with a limited sample size, which makes selection bias unavoidable. A certain degree of heterogeneity in terms of diagnosis and treatment might influence the results of the study. In addition, metastatic liver tumors are highly heterogeneous suggesting that there may be differences in pathologic response rates between different tumors. Thus, we chose the average pathologic response rate according to the method of Blazer et al. Finally, in this study we only performed internal validation since there was no external validation cohort. Because of this, the reliability of the model was suboptimal.

In conclusion, we found that preoperative characteristics such as DFI, metastatic liver tumor size and number, and $R A S$ mutational status were independent factors influencing pathologic response. The model we established for predicting pathologic response in patients with surgically treated CRLM after preoperative chemotherapy was highly sensitive and specific. We found that the model also performed well in subgroup analyses. This model could be very helpful for clinicians seeking to make better informed decisions regarding whether to perform surgery. A secondline therapy might be acceptable for patients with minor response. Thus, the current results may contribute to personalized care in the treatment of CRLM. However, future studies with larger sample sizes and multiple centers need to be conducted to verify the accuracy of the model proposed in this study.

\section{Acknowledgments}

Funding: This study was supported by grants (No. 81874143, No. 31971192) from the National Nature Science Foundation of China and the Beijing Natural Science Foundation (No. 7192035).

\section{Footnote}

Reporting Checklist: The authors have completed the Tripod reporting checklist. Available at https://dx.doi. org/10.21037/jgo-21-82

Data Sharing Statement: Available at https://dx.doi. org/10.21037/jgo-21-82

Peer Review File: Available at https://dx.doi.org/10.21037/ jgo-21-82

Conflicts of Interest: All authors have completed the ICMJE uniform disclosure form (available at https://dx.doi. org/10.21037/jgo-21-82). The authors have no conflicts of interest to declare.

Ethical Statement: The authors are accountable for all aspects of the work in ensuring that questions related to the accuracy or integrity of any part of the work are appropriately investigated and resolved. The study was conducted in accordance with the Declaration of Helsinki (as revised in 2013). The study was approved by Ethical Review Board committee of the Beijing Cancer Hospital and Institute (NO. 2015KT32) and informed consent was taken from all the patients.

Open Access Statement: This is an Open Access article distributed in accordance with the Creative Commons Attribution-NonCommercial-NoDerivs 4.0 International License (CC BY-NC-ND 4.0), which permits the noncommercial replication and distribution of the article with the strict proviso that no changes or edits are made and the original work is properly cited (including links to both the formal publication through the relevant DOI and the license). See: https://creativecommons.org/licenses/by-nc-nd/4.0/. 


\section{References}

1. Kopetz S, Chang GJ, Overman MJ, et al. Improved survival in metastatic colorectal cancer is associated with adoption of hepatic resection and improved chemotherapy. J Clin Oncol 2009;27:3677-83.

2. Mise Y, Aloia TA, Brudvik KW, et al. Parenchymal-sparing Hepatectomy in Colorectal Liver Metastasis Improves Salvageability and Survival. Ann Surg 2016;263:146-52.

3. Nordlinger B, Sorbye H, Glimelius B, et al. Perioperative FOLFOX4 chemotherapy and surgery versus surgery alone for resectable liver metastases from colorectal cancer (EORTC 40983): long-term results of a randomised, controlled, phase 3 trial. Lancet Oncol 2013;14:1208-15.

4. Xu D, Liu XF, Yan XL, et al. Survival prediction in patients with resectable colorectal liver metastases: Clinical risk scores and tumor response to chemotherapy. Oncol Lett 2017;14:8051-9.

5. Vigano L, Capussotti L, Barroso E, et al. Progression while receiving preoperative chemotherapy should not be an absolute contraindication to liver resection for colorectal metastases. Ann Surg Oncol 2012;19:2786-96.

6. Van Cutsem E, Cervantes A, Adam R, et al. ESMO consensus guidelines for the management of patients with metastatic colorectal cancer. Ann Oncol 2016;27:1386-422.

7. Eisenhauer EA, Therasse P, Bogaerts J, et al. New response evaluation criteria in solid tumours: revised RECIST guideline (version 1.1). Eur J Cancer 2009;45:228-47.

8. Blazer DG, 3rd, Kishi Y, Maru DM, et al. Pathologic response to preoperative chemotherapy: a new outcome end point after resection of hepatic colorectal metastases. J Clin Oncol 2008;26:5344-51.

9. Rubbia-Brandt L, Giostra E, Brezault C, et al. Importance of histological tumor response assessment in predicting the outcome in patients with colorectal liver metastases treated with neo-adjuvant chemotherapy followed by liver surgery. Ann Oncol 2007;18:299-304.

10. Margonis GA, Amini N, Andreatos N, et al. KRAS mutational status impacts pathologic response to prehepatectomy chemotherapy: a study from the International Genetic Consortium for Liver Metastases. HPB (Oxford) 2019;21:1527-34.

11. Serayssol C, Maulat C, Breibach F, et al. Predictive factors of histological response of colorectal liver metastases after neoadjuvant chemotherapy. World J Gastrointest Oncol 2019;11:295-309.

12. Wang Y, Yuan YF, Lin HC, et al. Pathologic response after preoperative therapy predicts prognosis of Chinese colorectal cancer patients with liver metastases. Chin J Cancer 2017;36:78.

13. Zimmitti G, Shindoh J, Mise Y, et al. RAS mutations predict radiologic and pathologic response in patients treated with chemotherapy before resection of colorectal liver metastases. Ann Surg Oncol 2015;22:834-42.

14. Chun YS, Vauthey JN, Boonsirikamchai P, et al. Association of computed tomography morphologic criteria with pathologic response and survival in patients treated with bevacizumab for colorectal liver metastases. JAMA 2009;302:2338-44.

15. Adam R, Wicherts DA, de Haas RJ, et al. Complete pathologic response after preoperative chemotherapy for colorectal liver metastases: myth or reality? J Clin Oncol 2008;26:1635-41.

16. Adam R, Wicherts DA, de Haas RJ, et al. Patients with initially unresectable colorectal liver metastases: is there a possibility of cure? J Clin Oncol 2009;27:1829-35.

17. Brouquet A, Zimmitti G, Kopetz S, et al. Multicenter validation study of pathologic response and tumor thickness at the tumor-normal liver interface as independent predictors of disease-free survival after preoperative chemotherapy and surgery for colorectal liver metastases. Cancer 2013;119:2778-88.

18. Shindoh J, Loyer EM, Kopetz S, et al. Optimal morphologic response to preoperative chemotherapy: an alternate outcome end point before resection of hepatic colorectal metastases. J Clin Oncol 2012;30:4566-72.

19. Xu D, Wang HW, Yan XL, et al. Sub-millimeter surgical margin is acceptable in patients with good tumor biology after liver resection for colorectal liver metastases. Eur J Surg Oncol 2019;45:1551-8.

20. Imai K, Allard MA, Benitez CC, et al. Early Recurrence After Hepatectomy for Colorectal Liver Metastases: What Optimal Definition and What Predictive Factors? Oncologist 2016;21:887-94.

21. Hutchins G, Southward K, Handley K, et al. Value of mismatch repair, KRAS, and BRAF mutations in predicting recurrence and benefits from chemotherapy in colorectal cancer. J Clin Oncol 2011;29:1261-70.

22. Schirripa M, Bergamo F, Cremolini C, et al. BRAF and RAS mutations as prognostic factors in metastatic colorectal cancer patients undergoing liver resection. Br J Cancer 2015;112:1921-8.

23. Sasaki K, Morioka D, Conci S, et al. The Tumor Burden Score: A New "Metro-ticket" Prognostic Tool For Colorectal Liver Metastases Based on Tumor Size and 
Number of Tumors. Ann Surg 2018;267:132-41.

24. Guglielmi AP, Sobrero AF. Second-line therapy for advanced colorectal cancer. Gastrointest Cancer Res 2007;1:57-63.

Cite this article as: $\mathrm{Xu} \mathrm{D}$, Wang YY, Yan XL, Li J, Wang $\mathrm{K}$, Xing BC. Development of a model to predict pathologic response to chemotherapy in patients with colorectal liver metastases. J Gastrointest Oncol 2021;12(4):1498-1508. doi: 10.21037/jgo-21-82
25. Gruenberger T, Arnold D, Rubbia-Brandt L. Pathologic response to bevacizumab-containing chemotherapy in patients with colorectal liver metastases and its correlation with survival. Surg Oncol 2012;21:309-15. 